\title{
Estimation à partir de mesures de réflectance spectrale du rayonnement photosynthétiquement actif absorbé par une culture de blé
}

\author{
F. Baret et A. Olioso \\ avec la collaboration technique de J.L. Luciani, J.F. Hanocq et J.C. Monterrot
}

INRA, station de bioclimatologie, BP 91, 84140 Montfavet, France

(reçu le 10 mars 1989; accepté le 18 juillet 1989)

Résumé - L'énergie photosynthétiquement active (PAR) absorbée par une culture détermine son potentiel de production de biomasse. Les réflectances spectrales sont fonctionnellement liées à la fraction d'énergie PAR absorbée ou interceptée par le couvert. Leur suivi dans le temps permet d'améliorer l'estimation de la production des cultures par télédétection. La relation entre l'indice de végétation (DN) combinant les réflectances spectrales rouge et proche infrarouge, et la fraction PAR absorbée par le couvert au cours de la journée $\left(\varepsilon_{j}\right)$, est analysée à partir de simulations du modèle de transfert radiatif SAIL. II apparaît que cette relation peut se mettre sous une forme simple :

$\varepsilon_{i}=0,94\left[1-((0,9-\mathrm{DN}) /(0,9-\mathrm{DN} s))^{\alpha}\right]$

où $\mathrm{DN}_{\mathrm{s}}$ représente la valeur de l'indice de végétation du sol nu et $\alpha$ dépend de la position du soleil et du port foliaire. Dans certaines conditions, la valeur de $\alpha$ est proche de l'unité et la relation entre $\varepsilon_{i}$ et DN devient linéaire.

Des mesures de l'indice de végétation ont été effectuées au sol sur des cultures de blé en phase d'implantation. Simultanément, la grandeur $\varepsilon_{i}$ a été estimée à partir de photographies hémisphériques prises au-dessus des cultures. Les relations expérimentales obtenues entre DN et $\varepsilon_{j}$ sont en bonne concordance avec la relation (a). L'exposant $\alpha$ varie de 1,33 à 2,22 suivant les conditions d'éclairement, rendant la relation entre DN et $\varepsilon_{i}$ non linéaire.

télédétection - réflectance - indice de végétation - blé - énergie photosynthétiquement active

Summary - Photosynthetically absorbed radiation by a wheat canopy estimated from spectral reflectance. Biomass production resulted from the conversion of photosynthetically active radiation (PAR) absorbed by the crop. Remotely sensed radiometrical data are functionally linked to the fraction of PAR energy absorbed by the canopy. Monitoring spectral reflectance of vegetation should contribute to improve the estimation of crop biomass production. The SAIL radiative transfer model is used to analyse the sensitivity of the relationship between the vegetation index $(D N)$ which combines red and near infrared reflectances and the PAR fraction absorbed daily by the canopy $\left(\varepsilon_{i}\right)$. For commodity, we have decomposed this relationship into two sub-relationships using a simple formalism to describe them with good accuracy:

- the ND-LAI (leaf area index) and

- the $L A I-\varepsilon_{i}$ relationships.

The combination of these two equations (equation (5)) and (equation (3)) leads to the following simple expression relating $\varepsilon_{i}$ to $N D$ :

$\varepsilon_{i}=0.94\left[1-\left((0.9-D N) /\left(0.9-D N_{s}\right)\right)^{\alpha}\right]$

$D N_{s}$ represents the $D N$ value for bare soil which varies with soil brightness (Fig. 3). $\alpha$ depends on sun position and leaf inclination (Fig. 5). However, in some conditions, $\alpha$ is very close to 1. and the relationship between $\varepsilon_{i}$ and $D N$ becomes linear.

DN measurements were performed at ground level on several wheat plots from emergence to stem elongation. Simultaneously, $\varepsilon_{i}$ was estimated from hemispherical photographs taken above the crop. Results show that experimental relationships between $D N$ and $\varepsilon_{i}$ are in accordance with the theoretical equation (a). The exponent $\alpha$ is in the range 1.33, 2.22 depending on irradiance conditions (Table III), making the relationship between DN and $\varepsilon_{j}$ nonlinear. In conclusion, the success of using spectral data to assess $\varepsilon_{j}$ is mainly influenced by our knowledge of soil optical properties $\left(N D_{s}\right)$ or by the potential development of improved algorithms to minimize soil effects on vegetation indices. 


\section{INTRODUCTION}

La production primaire d'un couvert végétal résulte de l'absorption du flux lumineux solaire incident et de sa transformation en biomasse par le système photosynthétique. Le rendement énergétique de ces processus peut être formalisé simplement à l'échelle instantanée par le produit de deux facteurs (Monteith, 1972; Varlet-Grancher et al., 1982). Le premier facteur, $\varepsilon_{i}$, est appelé efficience de l'absorption du rayonnement photosynthétiquement actif (PAR : 400-700 nm) par le couvert. II représente le rapport du PAR absorbé par le couvert au PAR incident. Le second facteur, $\varepsilon_{b}$, caractérise l'efficience de la transformation de la quantité d'énergie PAR absorbée, en matière sèche. La connaissance tout au long du cycle de la culture de ces deux termes, permet d'estimer la quantité de biomasse produite (Varlet-Grancher et al., 1982; Gosse et al., 1986; Green, 1987; Olioso, 1987; Garcia et al., 1988).

L'efficience $\varepsilon_{i}$ dépend principalement des propriétés optiques des feuilles actives dans le domaine PAR, de la réflectance de la strate inférieure non photosynthétiquement active (sol ou végétation sénescente), de la structure du couvert (indice foliaire, inclinaison des feuilles) et des caractéristiques de l'éclairement (position du soleil, importance du rayonnement diffus). $\mathrm{Ce}$ sont les mêmes processus de diffusion et d'absorption qui déterminent la réponse spectrale du couvert. II existe donc une relation fonctionnelle liée à la structure du couvert, entre le facteur $\varepsilon_{i}$ nécessaire à l'estimation de la production de biomasse, et les réflectances spectrales qui peuvent être mesurées par un capteur de télédétection dans le domaine des courtes longueurs d'onde. Cette relation a été mise en évidence expérimentalement par Ajai et al. (1984), Asrar et al. (1984), Hatfield et al. (1984), Daughtry (1988). Ces résultats ont été confirmés à partir de considérations théoriques par Sellers (1985), Kumar (1988), Baret \& Major (1988). Ces auteurs expriment généralement la réponse radiométrique du couvert sous forme d'indices de végétation, qui consistent en une combinaison particulière des réflectances dans les domaines spectraux rouge et proche infrarouge. Ces indices permettent de limiter l'influence de facteurs extérieurs au couvert végétal (géométrie de la visée et de l'éclairement, réflectance du sol, pente, ...).

L'analyse bibliographique révèle la variabilité des relations entre $\varepsilon_{i}$ et indice de végétation. Cet article a pour objectif l'analyse des facteurs de variation de ces relations, afin de mieux en cerner le domaine de validité. Pour ce faire, nous utiliserons dans un premier temps, un modèle de transfert radiatif de manière à analyser, du point de vue théorique, la sensibilité à différents facteurs de variation des relations entre $\varepsilon_{i}$ et indice de végétation. Dans un deuxième temps, nous comparerons les relations théoriques précédentes à des mesures réalisées sur des cultures de blé dans différentes conditions. Nous développerons à cette fin une méthode d'estimation de $\varepsilon_{i}$ basée sur l'utilisation de photographies hémisphériques.

\section{ANALYSE THÉORIQUE DE LA RELATION ENTRE $\varepsilon_{i}$ ET L'INDICE DE VÉGÉTATION}

\section{Méthode}

Nous avons utilisé le modèle de réflectance SAIL décrit par Verhoef $(1984,1985)$. Bien que relativement simple, il reproduit avec suffisamment de réalisme la réflectance bidirectionnelle de cultures homogènes. Ce modèle a été partiellement validé à plusieurs reprises (Le Master et al., 1980; Badhwar et al., 1985; Goel \& Deering, 1985; Rigal \& Terres, 1988). Les principales hypothèses sont :

- un couvert homogène horizontalement et infiniment étendu,

- des éléments du couvert lambertiens (feuilles et sol),

- des propriétés optiques identiques aux deux faces des feuilles,

- un azimut des feuilles aléatoire.

Nous avons simulé les réflectances dans le rouge $(R)$ et dans le proche infrarouge (PIR), de façon à calculer l'indice de végétation DN (différence normalisée), proposé par Rouse et al. (1974) :

$$
\mathrm{DN}=(\mathrm{PIR}-\mathrm{R}) /(\mathrm{PIR}+\mathrm{R})
$$

Cet indice très fréquemment utilisé, réalise un bon compromis entre simplicité et performances. Les différentes simulations ont été faites en supposant que les mesures radiométriques étaient effectuées au midi solaire.

Le modèle SAIL nous a permis de calculer à chaque instant, la fraction d'énergie photosynthétiquement active absorbée par le couvert. Cette quantité a été intégrée sur l'ensemble de la journée, en la convoluant à l'évolution du rayonnement incident instantané. Ceci est nécessaire afin de relier l'efficience de l'absorption, qui varie tout au long de la journée avec la position du soleil, à des mesures radiométriques instantanées réalisées à une heure donnée.

L'efficience $\varepsilon_{i}$ ainsi obtenue est compatible avec les données de rayonnement global journa- 
lier, fournies par la plupart des réseaux d'observations météorologiques. Dans ces calculs, nous avons considéré uniquement un rayonnement incident direct, proportionnel au cosinus de l'angle zénithal solaire.

La démarche adoptée consiste à simuler $\varepsilon_{j}$ et DN pour les différentes valeurs des variables d'entrée du modèle SAIL précisées par le Tableau I. Ces résultats théoriques servent de base à l'étude des relations entre $\varepsilon_{i}$ et DN.

\section{Analyse de la relation entre $D N$ et $\varepsilon_{\mathrm{i}}$}

Les simulations aboutissent à une relation statistique linéaire moyenne présentée par la Figure 1 et l'équation 2 :

$\varepsilon_{i}=1,328 \mathrm{DN}-0,308 \quad\left(\mathrm{R}^{2}=0,910 ; n=2016\right)$

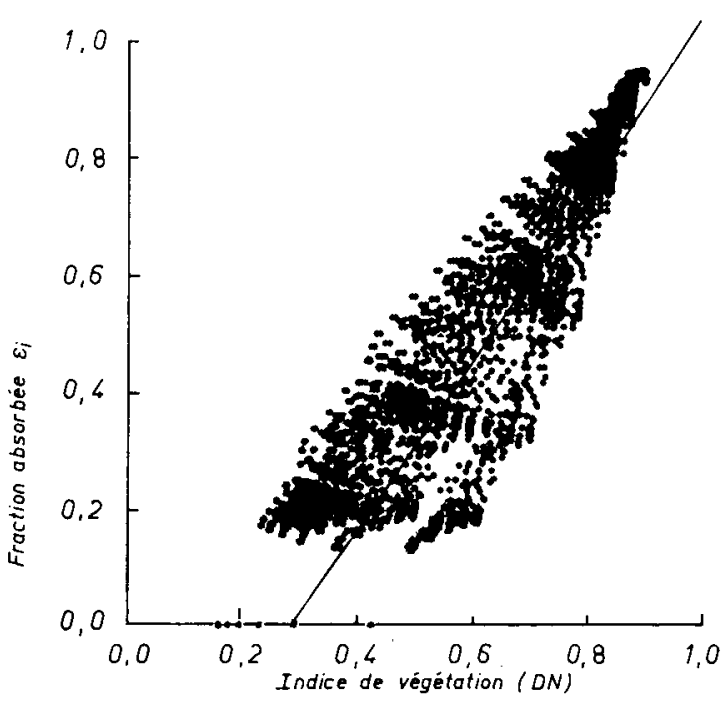

Fig. 1. Relation entre l'indice de végétation DN et l'efficience de l'absorption par le couvert du rayonnement photosynthétiquement actif $\left(\varepsilon_{i}\right)$. Les points correspondent aux simulations du modèle SAlL. La droite en trait plein correspond à la régression linéaire du nuage de points.
Cette relation est en assez bon accord avec celles proposées par Hatfield et al. (1984) ou Asrar et al. (1984), Sellers (1985), Daughtry (1988). Mais cet accord relatif ne doit pas masquer la variabilité importante existant autour de la droite moyenne, comme le montre la Figure 1. Nous allons à présent, chercher à préciser la nature et l'effet des facteurs perturbateurs, qui ne sont pas explicitement pris en compte dans l'approche statistique directe.

Afin de mieux décrire ces différents facteurs, nous avons décomposé la relation entre DN et $\varepsilon_{i}$ en deux sous-relations, en proposant pour chacune d'elles un formalisme simplifié :

- relation entre DN et l'indice foliaire,

- relation entre $\varepsilon_{i}$ et l'indice foliaire.

\section{Établissement d'une relation simplifiée entre DN et l'indice foliaire}

Plusieurs études expérimentales (Asrar et al., 1984; Kouassi, 1986) montrent que la relation entre DN et l'indice foliaire, suit une simple loi de Beer du type :

$$
D N=D_{\infty}+\left(D N_{s}-D N_{\infty}\right) e^{-K D N . L A l}
$$

avec

$\mathrm{DN}_{\infty}$ : valeur asymptotique de DN atteinte pour des indices foliaires tendant vers l'infini,

$\mathrm{DN}_{\mathrm{s}}$ : valeur de DN pour le sol nu (ou la strate sénescente sous-jacente),

$K_{D N}$ : coefficient d'extinction,

$L A I$ : indice foliaire.

Les paramètres $D_{\infty}$ et $K_{D N}$ dépendent ici des conditions d'éclairement et de l'inclinaison des feuilles. Pour chaque condition caractérisée par un angle zénithal solaire et un angle moyen d'in-

Tableau I. Paramètres et variables d'entrée du modèle de transfert radiatif SAlL utilisés pour nos simulations.

$\begin{array}{lccc}\text { Propriétés optiques } & R & P I R & P A R \\ \text { Réflectance feuilles } & 0,075 & 0,520 & 0,10 \\ \text { Transmittance feuilles } & 0,007 & 0,440 & 0,05\end{array}$

Réflectance sol (dans le rouge) (1) Indice foliaire

Angle d'inclinaison foliaire (2)

Angle zénithal solaire (3)

Orientation de la visée

$\begin{array}{lll}0,05 & 0,10 & 0,15 \\ 0,0 & 0,25 & 0,50 \\ 20^{\circ} & 30^{\circ} & 40^{\circ} \\ 10^{\circ} & 20^{\circ} & 30^{\circ} \\ \text { Visée verticale } & \end{array}$

$\begin{array}{lll}0,20 & 0,25 & 0,30 \\ 1,00 & 2,00 & 4,00 \\ 50^{\circ} & 60^{\circ} & 70^{\circ} \\ 40^{\circ} & 50^{\circ} & 60^{\circ}\end{array}$

8,00

$70^{\circ}$

$60^{\circ} \quad 70^{\circ}$

(1) La réflectance du sol dans le proche infrarouge est déduite de celle du rouge par l'équation de la droite du sol : PIR=1,16 $R+0,067$ (voir commentaires Fig. 3).

(2) Ceci correspond à l'angle moyen d'une fonction bêta de distribution des inclinaisons foliaires pour laquelle la variance est liée à la moyenne de manière à représenter correctement le cas d'une distribution sphérique (Goel \& Strebel, 1984).

(3) Pour le calcul de $\varepsilon_{i}$, ceci correspond à l'angle zénithal minimum (midi solaire). 
clinaison des feuilles, nous avons déterminé les valeurs de ces paramètres par ajustement non linéaire (Bouvier et al., 1985) de la fonction (3) aux DN simulés.

Le modèle simple (3) reproduit très correctement les résultats du modèle de transfert radiatif plus complexe SAIL $\left(R^{2}=0,995(2016)\right)$, et il permet d'analyser la dépendance de $D N_{\infty}$ et $K_{D N}$ à la position du soleil et à l'angle d'inclinaison des feuilles $\theta_{\mathrm{L}}$ (Fig. 2).

La valeur asymptotique $D_{\infty}$ varie de 0,89 à 0,94 (Fig. 2a). Ce paramètre ne semble pas très sensible à l'angle zénithal solaire ou à l'angle d'inclinaison des feuilles, sauf dans le cas de cultures plutöt érectophiles et de positions du soleil proches du zénith. La valeur moyenne de $\mathrm{DN}_{\infty}$ est proche de 0,90 . Le coefficient d'extinction $K_{D N}$, varie dans des proportions beaucoup plus importantes, de 0,5 à 2,1 avec une valeur moyenne proche de 1,2 (Fig. 2b). Pour des angles solaires zénithaux inférieurs à $50^{\circ}, \mathrm{K}_{\mathrm{DN}}$ diminue régulièrement quand l'inclinaison foliaire augmente, mais reste peu sensible à l'effet de la position du soleil. Au contraire, pour des angles zénithaux solaires supérieurs à $50^{\circ}, \mathrm{K}_{\mathrm{DN}}$ augmente très rapidement avec l'angle zénithal solaire, tout en restant très peu sensible à l'inclinaison foliaire.

Le modèle simplifié (3) permet également de calculer une expression analytique de la sensibilité aux propriétés optiques du sol sous-jacent :

$\mathrm{dDN} / \mathrm{dDNs}=e^{-\mathrm{KDN} \cdot L A l}$

La sensibilité de l'indice de végétation aux propriétés optiques du sol sera donc d'autant plus

(a)

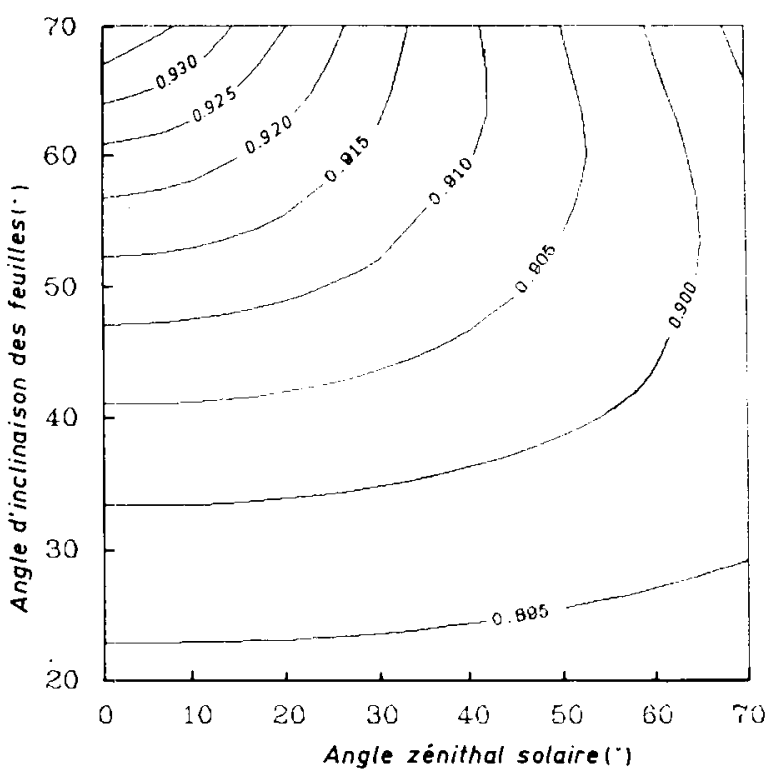

importante que l'indice foliaire ou le coefficient d'extinction seront faibles. Ce sera le cas pour des cultures érectophiles avec une mesure radiométrique réalisée quand le soleil est au plus haut. Ce sera également le cas pour des sols sombres qui présentent une sensibilité maximale à des variations de propriétés optiques (Fig. 3). Ces propriétés optiques dépendent du type de sol, et pour un type de sol donné, de sa rugosité et de son humidité de surface.

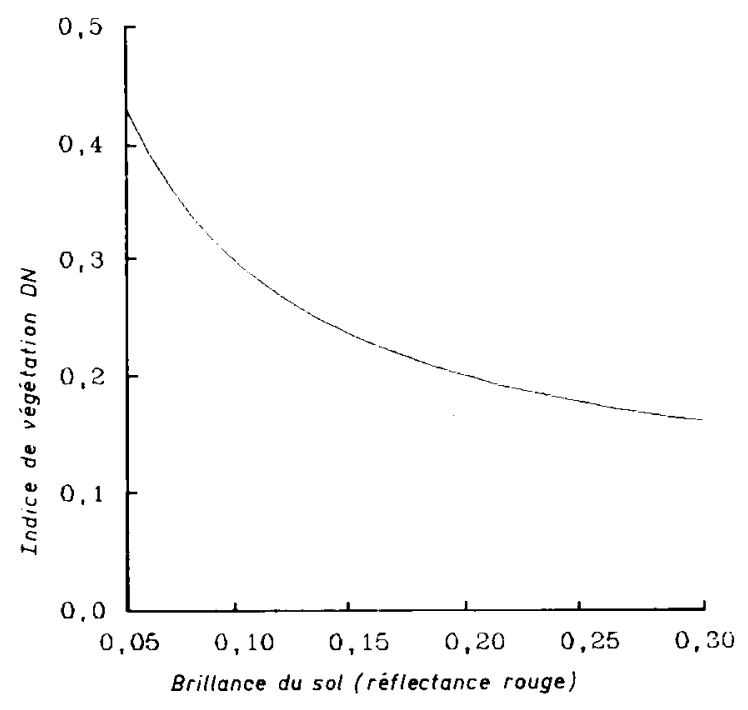

Fig. 3 Effet de la brillance du sol (représentée par sa réflectance dans le rouge (R)) sur la valeur de son indice de végétation DN. Ces résultats correspondent au sol de la station expérimentale de Montfavet ayant comme droite du sol (relation linéaire entre sa réflectance rouge et sa réflectance proche infrarouge (PIR)) : PIR=1,16 R+0,067 (Baret, 1986).

Fig. 2. (a) : Influence de l'angle zénithal solaire $\left(\theta_{s}\right)$ et de l'angle moyen d'inclinaison des feuilles $\left(\theta_{L}\right)$ sur $D N_{\infty}$; (b) : idem pour $K_{D N}$. 


\section{Établissement d'une relation simplifiée entre $\varepsilon_{i}$ et l'indice foliaire}

Nous avons employé un formalisme classiquement utilisé pour décrire les résultats de diverses études expérimentales (Monteith, 1981; Asrar et al., 1984) :

$$
\varepsilon_{i}=\varepsilon_{i \infty}\left(1-e^{-K \varepsilon i . L A l}\right)
$$

avec

$\varepsilon_{i \infty}$ : valeur asymptotique de $\varepsilon_{i}$ pour des indices foliaires tendant vers l'infini,

$\mathrm{K}_{\varepsilon i}$ : coefficient d'extinction.

Les paramètres $\varepsilon_{i \infty}$ et $K_{\varepsilon i}$ dépendant eux aussi des conditions d'éclairement et de l'orientation des feuilles. Ils ont été déterminés en utilisant la même démarche que celle utilisée au paragraphe précédent pour $\mathrm{DN}_{\infty}$ et $\mathrm{K}_{\mathrm{DN}}$.

Ce modèle simplifié est également en très bon accord avec le modèle SAIL ( $R^{2}=0,998$ (2016)). L'équation (5) ne prend pas en compte l'effet des propriétés optiques du sol tout en restant très performante. L'influence des propriétés optiques du sol sur la valeur de la fraction absorbée par le couvert dans le domaine PAR, est en effet peu importante.

La Figure 4 montre la sensibilité des paramètres $\varepsilon_{i \infty}$ et $K_{\varepsilon i}$ à l'angle zénithal solaire au midi solaire $\left(\theta_{12}, \varepsilon_{i}\right.$ étant intégré sur la journée) et à l'angle d'inclinaison des feuilles. La valeur asymptotique $\varepsilon_{i_{\infty}}$ varie dans de faibles proportions de 0,93 à 0,95 , avec une valeur moyenne proche de 0,94 (Fig. 4a). En revanche, le coeffi- cient d'extinction $\mathrm{K}_{\varepsilon i}$ varie dans des proportions assez semblables à celles de $\mathrm{K}_{\mathrm{DN}}$, de 0,6 à 2,2 avec une valeur moyenne proche de 1,0 (Fig. $4 b)$. Comme $K_{D N}, K_{\varepsilon i}$ diminue régulièrement quand l'inclinaison foliaire augmente pour des angles zénithaux au midi solaire inférieurs à $30^{\circ}$. II est alors moins sensible à l'angle zénithal solaire. En revanche, pour des angles zénithaux solaires supérieurs à $30^{\circ}, \mathrm{K}_{\varepsilon}$ augmente rapidement avec l'angle zénithal solaire $\theta_{12}$, et est alors peu sensible à l'inclinaison des feuilles.

\section{Établissement d'une relation entre DN et $\varepsilon_{i}$}

Le couplage des deux modèles simplifiés précédents permet de construire une relation analytique entre $\mathrm{DN}$ et $\varepsilon_{i}$ :

$\varepsilon_{i}=\varepsilon_{i \infty}\left[1-\left(\left(\mathrm{DN}-\mathrm{DN}_{\infty}\right) /\left(\mathrm{DN}_{\mathrm{s}}-\mathrm{DN}_{\infty}\right)\right)^{\left.\mathrm{K}_{\varepsilon} / \mathrm{KDN}\right](6)}\right.$

Cette équation formalise l'effet des propriétés optiques du sol sur $\varepsilon_{i}$ par l'intermédiaire du terme $\mathrm{DN}_{\mathrm{s}}$. La dépendance de $\mathrm{DN}_{\infty}$ à la réflectance du sol étant plus accentuée pour les sols sombres (Fig. 3), la sensibilité de la relation entre $\varepsilon_{i}$ et DN aux propriétés optiques du sol sera importante dans ce cas. L'équation (6) montre également que la linéarité de la relation n'est pas stricte, et dépend du rapport des coefficients d'extinction $\mathrm{K}_{\mathrm{e} i} / \mathrm{K}_{\mathrm{DN}}$. Les remarques effectuées aux deux paragraphes précédents sur le sens de variation et les valeurs des deux coefficients $K_{\varepsilon i}$ et $K_{D N}$, révèlent une certaine similitude de leur comportement. II semble possible en première approxi- (a)

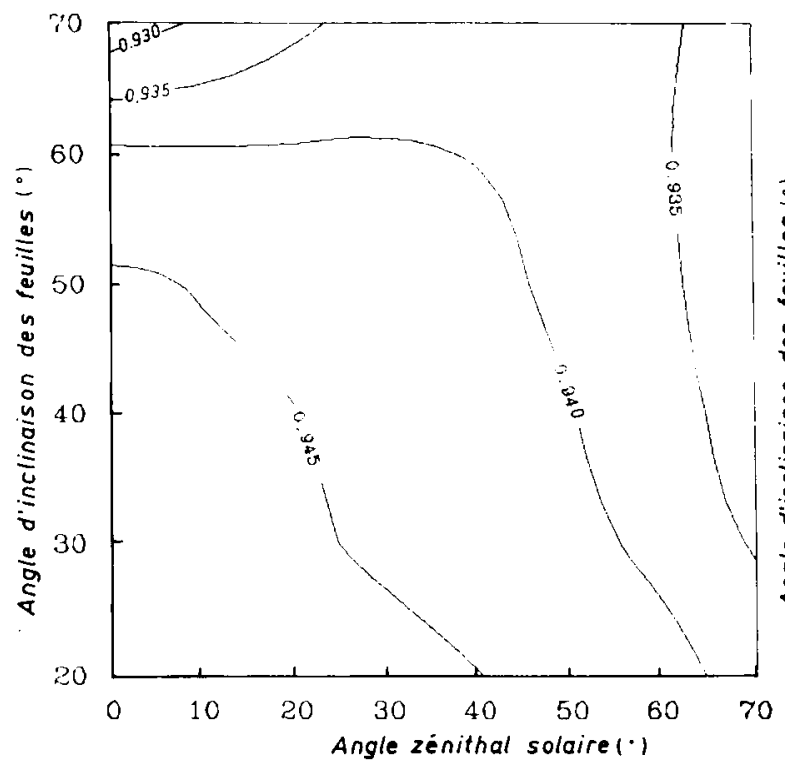

(b)

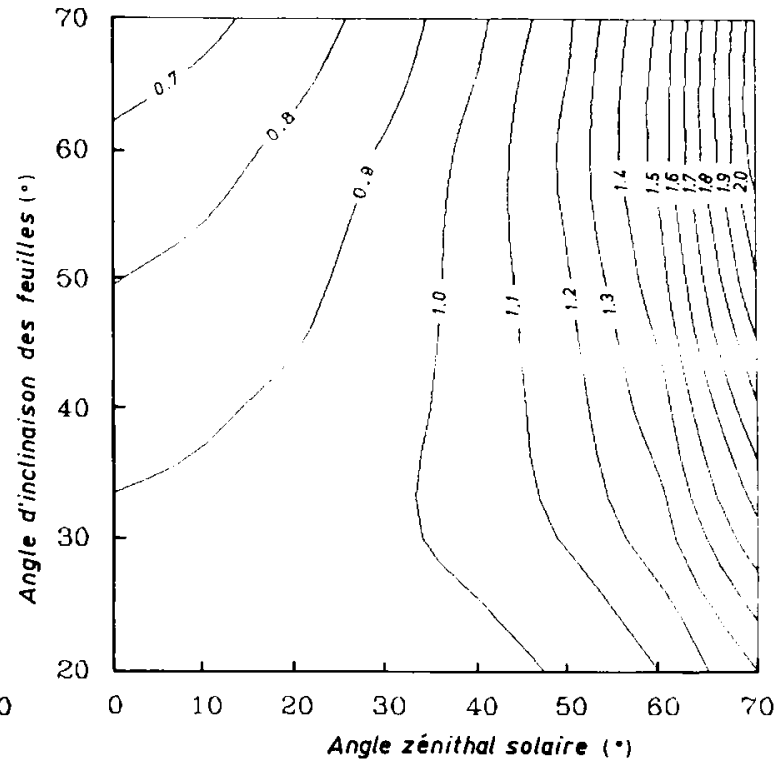

Fig. 4. (a) Influence de l'angle zénithal solaire au midi solaire $\left(\theta_{12}, \varepsilon_{i}\right.$, étant intégré sur la journée) et de l'angle d'inclinaison foliaire $\theta_{\mathrm{L}}$ sur $\varepsilon_{i \infty o}$. (b) Idem pour $\mathrm{K}_{\varepsilon i}$. 
mation, de poser $\mathrm{K}_{\mathrm{DN}}=\mathrm{K}_{\varepsilon i}$ quand les mesures radiométriques sont effectuées au midi solaire $\left(\theta_{s}=\theta_{12}\right)$. Cette approximation n'est exactement vérifiée que pour un nombre limité de cas. Ils correspondent (Fig. 5), à des angles moyens d'inclinaison foliaire proches de $60^{\circ}$, et ce, relativement indépendamment de l'angle zénithal solaire. Les quelques rares études s'étant intéressées à la mesure de la fonction de distribution des angles foliaires de cultures de blé (Le Master et al., 1980; Goel \& Strebel, 1984; Baret, 1986), révèlent des angles moyens d'inclinaison compris entre $40^{\circ}$ et $70^{\circ}$. Ils peuvent donc conduire à des différences significatives entre $K_{D N}$ et $K_{\varepsilon}$.

En supposant $K_{D N}$ et $K_{\varepsilon i}$ identiques, la relation (6) se simplifie et devient linéaire. Les paramètres $\varepsilon_{i \infty}$ et $\mathrm{DN}_{\infty}$ sont peu sensibles à la position du soleil, ainsi qu'à l'inclinaison des feuilles. Nous pouvons les confondre à leur valeur moyenne, et l'équation devient alors :

$$
\varepsilon_{i}=0,94\left(\left(\mathrm{DN}-\mathrm{DN}_{\mathrm{s}}\right) /\left(0,9-\mathrm{DN}_{\mathrm{s}}\right)\right)
$$

L'application de l'équation (7) aboutit à une estimation correcte de $\varepsilon_{i}$ à partir de $\mathrm{DN}$ en comparaison des simulations du modèle SAIL $\left(R^{2}=0,964\right.$ (2016), Fig. 6). Cette relation linéaire prend en compte explicitement les propriétés optiques du sol contrairement à celles généralement proposées dans la littérature.

Au terme de cette première partie, nous avons établi une expression simple de la relation entre $\varepsilon_{i}$ et DN. Dans la partie suivante, nous en testerons la validité par rapport à nos résultats éxpérimentaux établis sur des cultures de blé en phase d'implantation. Dans ce cas, la structure en rangs ne correspond plus aux hypothèses d'homogénéité du couvert formulées au travers du modèle SAIL.

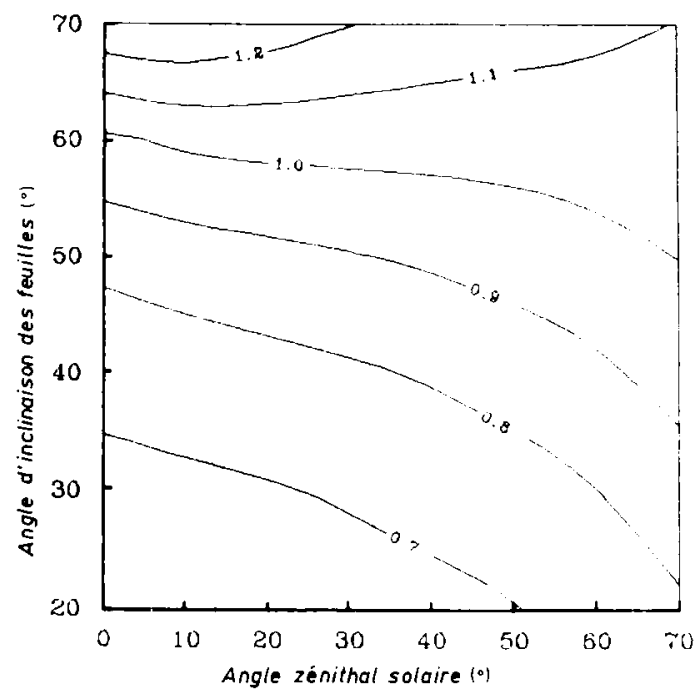

Fig. 5. Influence de l'angle solaire zénithal $\left(\theta_{12}\right)$ et de l'angle d'inclinaison $\left(\theta_{L}\right)$ sur le rapport $K_{\varepsilon} / K_{D N}$.

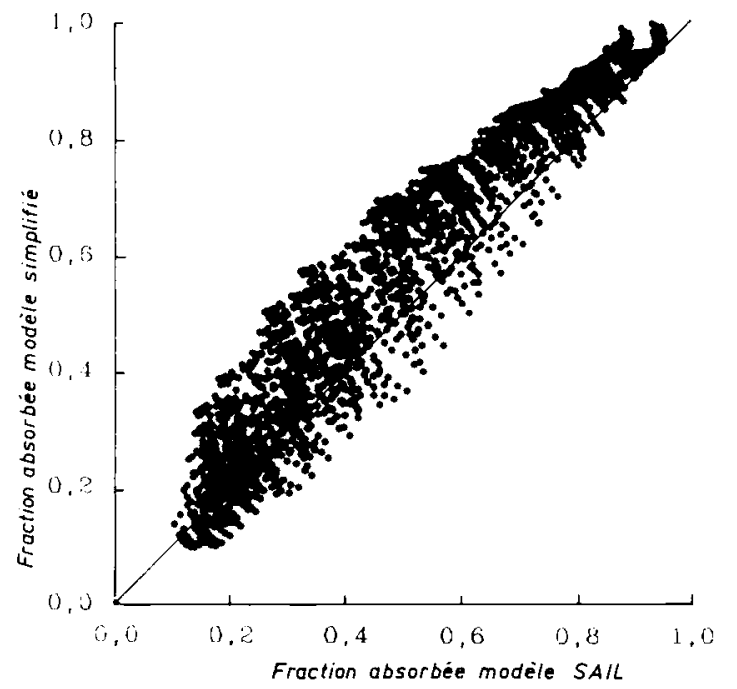

Fig. 6. Relation entre $\varepsilon_{i}$ estimé à partir de l'équation (7) et $\varepsilon_{i}$ estimé à partir des simulations du modèle SAIL.

\section{MISE EN ÉVIDENCE EXPÉRIMENTALE DE LA RELATION ENTRE $\varepsilon_{i}$ ET DN}

\section{L'expérimentation}

\section{Le site expérimental et les cultures}

L'expérimentation s'est déroulée sur le domaine expérimental d'Avignon-Montfavet ( $4^{\circ} 51^{\prime}$ long. $E$; $43^{\circ} 55^{\prime}$ lat. N), situé dans la plaine alluviale de la basse vallée du Rhône. Les semis de blé (variété Fidel) ont été réalisés à 3 époques différentes: 01 octobre 1986 (Fidel 1), 28 novembre 1986 (Fidel 2) et 27 février 1987 (Fidel 3). Une deuxième variété (Arminda) de port foliaire plus dressé a été semée le 28 novembre 1986 (Arminda 2). La densité à la levée variait entre 300 et 400 plantes $/ \mathrm{m}^{2}$. Les rangs étaient orientés nord-sud et écartés de $0,17 \mathrm{~m}$. II faut noter que la première date de semis fut fortement atteinte au cours de la montaison par différentes maladies et parasites.

\section{Les mesures radiométriques}

L'indice de végétation $D N$ est calculé à partir des réflectances rouge $(610-680 \mathrm{~nm})$ et proche infrarouge $(790-890 \mathrm{~nm})$ mesurées à l'aide d'un réflectomètre CIMEL (Guyot et al., 1984). Cet appareil est constitué de deux têtes de mesure : une tête de mesure de l'éclairement équipée d'objectifs diffusants ( $180^{\circ}$ d'ouverture) et une tête de mesure de la luminance équipée d'objectifs de $12^{\circ}$ d'ouverture. Le réflectomètre est fixé à $5 \mathrm{~m}$ de hauteur. La surface visée est d'environ $0,85 \mathrm{~m}^{2}$ et un minimum de 6 répétitions sont 
effectuées sur chaque traitement. L 'étalonnage du réflectomètre est réalisé en visant une surface de référence de réflectance connue. Simultanément, des mesures de rayonnement global et de rayonnement diffus renseignent sur les conditions d'éclairement. Une à deux séries de mesures sont enregistrées chaque semaine au midi solaire, à partir de la levée de la première date de semis jusqu'à la récolte. Seules les mesures correspondant à des conditions où le rapport diffus/direct est inférieur à $40 \%$ (ciel dégagé) ont été retenues. Une parcelle de sol nu est intercalée entre les traitements et fournit les valeurs de $\mathrm{DN}_{\mathrm{s}}$.

La Figure 7 montre l'évolution de l'indice de végétation pour les différents traitements. Les valeurs de DN correspondant aux mesures de $\varepsilon_{i}$ sont reportées au Tableau II ainsi que les valeurs de sol nu associées $\left(\mathrm{DN}_{\mathrm{s}}\right)$.

\section{Les mesures de $\varepsilon_{i}$ : utilisation de photogra- phies hémisphériques}

La détermination de la fraction du rayonnement photosynthétiquement actif absorbée par un couvert est réalisée classiquement par des méthodes de bilan radiatif (Varlet-Grancher et al., 1982). Ces méthodes posent différents problèmes pour des couverts en phase d'installation ayant un développement vertical faible. Nous
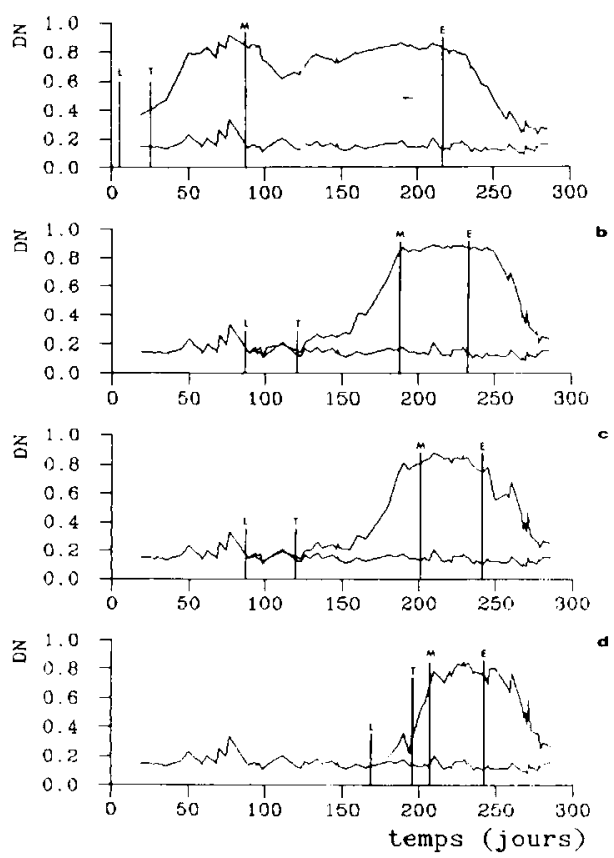

Fig. 7. Evolution de l'indice de végétation (DN) pour les 4 traitements étudiés ( $a$ : Fidel $1 ; b$ : Fidel 2; $c$ : Arminda 2; $d$ : Fidel 3). Le temps est exprimé en jours comptés à partir du 1 octobre 1986. Les différentes dates de stade phénologique sont indiquées ( $L$ : Levée; $T$ : Début tallage; $M$ : Stade épi à $1 \mathrm{~cm}$; $\mathrm{E}$ : Epiaison). Pour chaque graphique, la courbe du haut est celle du traitement considéré et la courbe du bas correspond aux mesures du sol nu. avons développé une méthodologie basée sur l'utilisation de photographies hémisphériques réalisées par dessus le couvert avec un film infrarouge couleur.

Les photographies hémisphériques ont été utilisées par différents auteurs (Bonhomme \& Chartier, 1972; Ducrey, 1975a, 1975b) pour obtenir une information directionnelle sur la transmission du rayonnement solaire sous culture ou sous forêt. Elles nous permettent de calculer la fraction interceptée par la végétation pour chaque direction d'incidence. La distinction entre les éléments verts photosynthétiquement actifs et le sol, ou la végétation sénescente, est réalisée en combinant les images rouge et proche infrarouge extraites de la photographie, sous la forme d'un «indice de végétation» (Olioso, 1987). Une étude particulière, également réalisée à partir du modèle SAIL, montre que dans le domaine PAR, et pour des feuilles vertes, la fraction interceptée est très peu différente de la fraction absorbée : $94 \%$ du rayonnement intercepté est effectivement absorbé par le couvert. La bonne adéquation de ces deux grandeurs est liée à la très forte absorption des feuilles vertes dans le domaine PAR ( $85 \%$ dans ce cas particulier).

Dans ces conditions, l'utilisation de photographies hémisphériques infrarouge couleur permet de reconstruire l'évolution diurne de l'absorption du PAR par le couvert, en convoluant la fraction absorbée dans la direction d'incidence du soleil, à la quantité d'énergie incidente correspondante. Cette méthode, contrairement à celle basée sur le bilan radiatif, ne dépend pas des conditions d'éclairement au moment de la mesure. Elle dépend seulement de l'architecture du couvert, ce qui nous permet de calculer $\varepsilon_{i}$ pour différentes conditions d'éclairement. Nous avons réalisé ce calcul dans 5 cas extrêmes :

- Pour des conditions d'éclairement diffus homogène uniquement,

- Pour des conditions d'éclairement directionnel uniquement en distinguant alors 4 cas :

- Au moment du solstice d'hiver (soleil bas sur I'horizon) $\left(\theta_{12}=67^{\circ}\right)$,

- A l'équinoxe $\left(\theta_{12}=44^{\circ}\right)$,

- Au moment du solstice d'été (soleil relativement haut) $\left(\theta_{12}=20^{\circ}\right)$,

- En supposant l'angle solaire $\theta_{12}$ égal à l'angle du soleil au moment de la mesure radiométrique (effectuée au midi solaire, cf. Tableau II). Ce cas correspond aux conditions réelles de nos expérimentations.

Nous avons utilisé un objectif "fish-eye" Nikkor $(7,5 \mathrm{~mm}$ de focale), placé à environ $0,10 \mathrm{~m}$ au-dessus du couvert, et des films infrarouges couleurs Ektachrome 2236. Afin de prendre en 
Tableau II. Valeurs d'indice de végétation et valeurs d' $\varepsilon_{i}$ estimées à partir des photographies hémisphériques pour différentes conditions d'éclairement définies ci-dessus.

\begin{tabular}{|c|c|c|c|c|c|c|c|c|c|c|}
\hline \multirow{2}{*}{ Traitement } & \multirow{2}{*}{ Date } & \multirow{2}{*}{$\begin{array}{c}T^{\circ} \\
\text { depuis le } \\
01 / 10 / 86 \\
\text { (base } 0^{\circ} \mathrm{C} \text { ) }\end{array}$} & \multicolumn{3}{|c|}{$\begin{array}{l}\text { Mesures } \\
\text { radiométriques }\end{array}$} & \multirow[b]{2}{*}{ Diffus } & \multicolumn{4}{|c|}{$\begin{array}{c}\varepsilon_{i}, \text { estimé par photographies } \\
\text { hémisphériques }\end{array}$} \\
\hline & & & $\theta_{s}\left(^{*}\right)$ & $D N$ & $D N_{s}$ & & $\begin{array}{l}\text { Sols. } \\
\text { hiver }\end{array}$ & Equinoxe & $\begin{array}{l}\text { Sols. } \\
\text { été }\end{array}$ & $\theta_{12}$ \\
\hline \multirow[t]{6}{*}{ Fidel 1} & $24-10-86$ & 395 & 56 & 0,383 & 0,148 & 0,330 & 0,470 & 0,305 & 0,250 & 0,391 \\
\hline & $12-11-86$ & 611 & 61 & 0,581 & 0,152 & 0,688 & 0,782 & 0,677 & 0,643 & 0,755 \\
\hline & $19-02-87$ & 1219 & 55 & 0,756 & 0,155 & 0,875 & 0,933 & 0,907 & 0,863 & 0,919 \\
\hline & $04-03-87$ & 1319 & 50 & 0,752 & 0,116 & 0,876 & 0,936 & 0,899 & 0,866 & 0,906 \\
\hline & $18-03-87$ & 1397 & 45 & 0,807 & 0,154 & 0,826 & 0,908 & 0,876 & 0,828 & 0,877 \\
\hline & $15-04-87$ & 1679 & 34 & 0,837 & 0,142 & 0,894 & 0,927 & 0,913 & 0,894 & 0,905 \\
\hline \multirow[t]{3}{*}{ Fidel 2} & $19-02-87$ & 1219 & 55 & 0,252 & 0,155 & 0,271 & 0,276 & 0,217 & 0,280 & 0,245 \\
\hline & $04-03-87$ & 1312 & 50 & 0,272 & 0,116 & 0,395 & 0,438 & 0,347 & 0,340 & 0,410 \\
\hline & $02-04-87$ & 1530 & 39 & 0,707 & 0,165 & 0,863 & 0,930 & 0,891 & 0,847 & 0,882 \\
\hline \multirow[t]{5}{*}{ Arminda 2} & $19-02-87$ & 1219 & 55 & 0,222 & 0,155 & 0,213 & 0,211 & 0,164 & 0,141 & 0,187 \\
\hline & $04-03-87$ & 1312 & 50 & 0,212 & 0,116 & 0,188 & 0,201 & 0,158 & 0,132 & 0,169 \\
\hline & $18-03-87$ & 1403 & 45 & 0,312 & 0,154 & 0,319 & 0,347 & 0,254 & 0,251 & 0,258 \\
\hline & $02 \cdot 04-87$ & 1530 & 39 & 0,584 & 0,165 & 0,706 & 0,817 & 0,691 & 0,662 & 0,684 \\
\hline & $15-04-87$ & 1679 & 34 & 0,777 & 0,142 & 0,901 & 0,934 & 0,920 & 0,903 & 0,913 \\
\hline \multirow[t]{3}{*}{ Fidel 3} & $02-04-87$ & 1530 & 39 & 0,221 & 0,165 & 0,185 & 0,218 & 0,159 & 0,136 & 0,154 \\
\hline & $15-04-87$ & 1679 & 34 & 0,285 & 0,142 & 0,318 & 0,392 & 0,275 & 0,258 & 0,268 \\
\hline & $28-04-87$ & 1862 & 30 & 0,566 & 0,130 & 0,552 & 0,709 & 0,543 & 0,490 & 0,512 \\
\hline
\end{tabular}

(*) Angle solaire zénithal au moment de la mesure radiométrique.

compte la variabilité et la structure en rang du couvert, quatre répétitions de couple de photographies sur le rang et sur l'interrang sont effectuées. Compte tenu de l'orientation nord-sud des rangs et en supposant une distribution azimutale des feuilles aléatoire, chaque photographie représente la composition de 4 quadrants identiques. Ceux-ci forment alors 32 pseudo-répétitions sur lesquelles les moyennes d'interception directionnelle sont calculées par pas de $10^{\circ}$ en azimut et $10^{\circ}$ en zénith. La numérisation des films photographiques est effectuée à l'aide d'une caméra CCD de résolution $500 \times 500$ équipée de filtres permettant d'extraire séparément l'image rouge et l'image infrarouge. La caméra est reliée à un micro-ordinateur par l'intermédiaire d'une carte de numérisation. Après combinaison des images numériques rouge et infrarouge, un seuillage assure la séparation entre la partie végétation verte et les autres éléments. L'indétermination sur la valeur seuil conduit à une imprécision de l'ordre de $10 \%$ pour chaque photographie.

Les dates des différentes photographies ainsi que les valeurs correspondantes de $\varepsilon_{i}$ pour les 5 conditions d'éclairement précédemment définies sont reportées au Tableau II.

\section{RÉSULTATS ET DISCUSSION}

La Figure 7 montre que l'indice de végétation DN est sensible à l'état du sol. Les pics notés en début et en fin de cycle correspondent à des périodes pendant lesquelles la surface du sol est humide (valeurs de $\mathrm{DN}_{\mathrm{s}}$ plus fortes). La valeur maximale de $\mathrm{DN}$ mesurée ( $\mathrm{DN}=0,89$ (Fig. 7)) est proche de la valeur asymptotique moyenne calculée par le modèle SAIL ( $\left.\mathrm{DN}_{\infty}=0,90\right)$. De même, les valeurs maximales observées de $\varepsilon_{i}\left(\varepsilon_{i}=0,93\right.$ (Tableau II) sont proches de la valeur asymptotique fournie par le modèle SAIL $\left(\varepsilon_{i_{\infty}}=0,94\right)$. Ces valeurs de $\mathrm{DN}_{\infty}$ et $\varepsilon_{i \infty}$ sont également en bonne concordance avec les résultats d'Asrar et al. (1984). Pour tester le modèle (6), nous avons fixé les paramètres $D N_{\infty}$ et $\varepsilon_{i_{\infty}}$ respectivement à 0,90 et 0,94 . Nous avons calé le paramètre $\mathrm{K}_{\varepsilon i} / \mathrm{K}_{\mathrm{DN}}$ en utilisant les programmes d'ajustements non linéaires développés par Bouvier et al. (1985), basé sur la maximisation de la fonction de vraisemblance. Ce critère nous permet ainsi de comparer statistiquement des ajustements en confrontant le rapport de leur maximum de vraisemblance à une loi de $\chi^{2}$. Le Tableau III présente les résultats de différents ajustements. Dans le premier cas (a), l'ajustement est réalisé 
Tableau III. Comparaison des différents modèles testés.

$(*)$

Modèles utilisés

Conditions

d'éclairement (a)

$\varepsilon_{i \infty} \quad \mathrm{K}_{\varepsilon i} / \mathrm{K}_{D N}$

(b)

$\overline{\varepsilon_{i \infty}} \quad \mathrm{K}_{\varepsilon i} / \mathrm{K}_{D N}$

(c)

$\overline{\varepsilon_{i \infty}} \quad \mathrm{K}_{\varepsilon /} / K_{D N}$

\begin{tabular}{|c|c|c|c|c|c|c|}
\hline Diffus & 0,94 & 1,599 & 0,94 & 1,654 & 0,94 & 1,00 \\
\hline Solstice d'hiver & 0,94 & 2,218 & 0,94 & 1,654 & 0,94 & 1,00 \\
\hline Equinoxe & 0,94 & 1,503 & 0,94 & 1,654 & 0,94 & 1,00 \\
\hline Solstice d'été & 0,94 & 1,332 & 0,94 & 1,654 & 0,94 & 1,00 \\
\hline$\theta_{12}$ & 0,94 & 1,619 & 0,94 & 1,654 & 0,94 & 1,00 \\
\hline$\left(^{\star \star}\right) 2 \lg V$ & \multicolumn{2}{|c|}{241,46} & \multicolumn{2}{|c|}{ 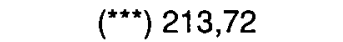 } & \multicolumn{2}{|c|}{$\left({ }^{\star \star *}\right) 168,19$} \\
\hline
\end{tabular}

" : Les modèles testés correspondent tous à l'équation (6) pour laquelle on a posé $\mathrm{DN}_{\infty}=0,9$ et $\varepsilon_{i \infty}=0,94$; (a) On distingue les différentes conditions d'éclairement pour ajuster $K_{\varepsilon i} / K_{D N}$ (b) On ne distingue pas les differentes conditions d'éclairement pour ajuster $K_{\varepsilon i} / K_{D N} ;(c)$ On fixe $K_{\varepsilon i} / K_{D N} a ̀ 1,00$ : modèle équivalent à l'équation linéaire (7).

** $V$ : Vraisemblance

*** : significativement différent de (a) $(P=0,10)$.

séparément pour chacune des 5 conditions d'éclairement définies pour le calcul de $\varepsilon_{i}$. Le second cas (b) correspond à un ajustement global sans distinction des conditions d'éclairement.

Le rapport $K_{\varepsilon i} / K_{D N}$ dépend des conditions d'éclairement prises en compte pour calculer $\varepsilon_{i}$, comme le montrent les valeurs obtenues pour l'ajustement (a) et la différence significative qui existe entre (a) et (b). Le modèle linéaire (c) pour lequel on fixe $\mathrm{K}_{\varepsilon i} / \mathrm{K}_{\mathrm{DN}}$ à 1,00 est significativement différent des modèles plus généraux (a) ou (b). La non linéarité de la relation entre $\mathrm{DN}$ et $\varepsilon_{i}$ est d'autant plus forte que les faibles élévations solaires prennent du poids (solstice d'hiver, diffus $\theta_{12}$ ). Cela s'accorde bien avec les résultats de l'analyse théorique de la première partie (Fig. 5), mais s'oppose en première analyse aux résultats expérimentaux d'Hattield et al. (1984) ou Asrar et al. (1984), et Daughtry (1988). En fait, ces études ont principalement été réalisées pour des latitudes faibles ( $\theta_{12}$ faible) et révèlent tout de même une tendance à la non linéarité.

En revanche, nos données expérimentales ne nous permettent pas de mettre en évidence l'effet du port foliaire sur la relation entre DN et $\varepsilon_{i}$. La Figure 7 révèle des variations de port foliaire visibles à partir de l'évolution de DN. On remarque que pour le semis précoce (Fidel 1), le redressement au stade "épi $1 \mathrm{~cm}$ " s'accompagne d'une diminution de l'indice de végétation. De même, la comparaison des évolutions de DN des variétés Fidel et Arminda en $2^{\theta}$ date de semis révèle le port plus érectophile d'Arminda. Malheureusement, le faible nombre de points expérimentaux de certains traitements, ainsi que la liaison forte entre stade phénologique, indice foliaire et port foliaire ne nous permet pas de conclure à un effet du port foliaire.

Les valeurs expérimentales du rapport $\mathrm{K}_{\varepsilon i} / \mathrm{K}_{\mathrm{DN}}$ sont supérieures à celles obtenues à partir de nos simulations. L'effet rang, non pris en compte par le modèle SAIL qui suppose le couvert homogène dans le plan horizontal, explique sans doute une partie des divergences observées. De plus les ajustements réalisés à partir des simulations du modèle SAlL supposent des variations de l'indice foliaire indépendantes de la position du soleil, alors qu'en conditions expérimentales ces évolutions sont simultanées.

Le modèle non linéaire (8) semble donc le mieux adapté pour décrire la relation entre $\mathrm{DN}$ et $\varepsilon_{i}:$

$$
\varepsilon_{i}=0,94\left[1-\left((0,9-\mathrm{DN}) /\left(0,9-\mathrm{DN}_{\mathrm{s}}\right)\right)^{\mathrm{K} \varepsilon i / \mathrm{KDN}](8)}\right.
$$

La Figure 8 montre la bonne adéquation entre $\varepsilon_{i}$ estimé par ce modèle et $\varepsilon_{i}$ mesuré. L'écart type résiduel sur $\varepsilon_{i}$ est inférieur à 0,06 . Celui du modèle linéaire correspondant est de 0,10.

\section{CONCLUSION}

L'analyse théorique réalisée à partir de simulations du modèle SAIL montre que la relation entre $\varepsilon_{i}$ et $\mathrm{DN}$ est sous la dépendance des conditions d'éclairement au cours de la journée (nécessaires au calcul de la fraction absorbée) ainsi que de celles présentes au moment de la mesure radiométrique. Ces paramètres sont parfaitement contrôlables ou mesurables. La relation entre $\varepsilon_{i}$ et $\mathrm{DN}$ dépend aussi des propriétés 


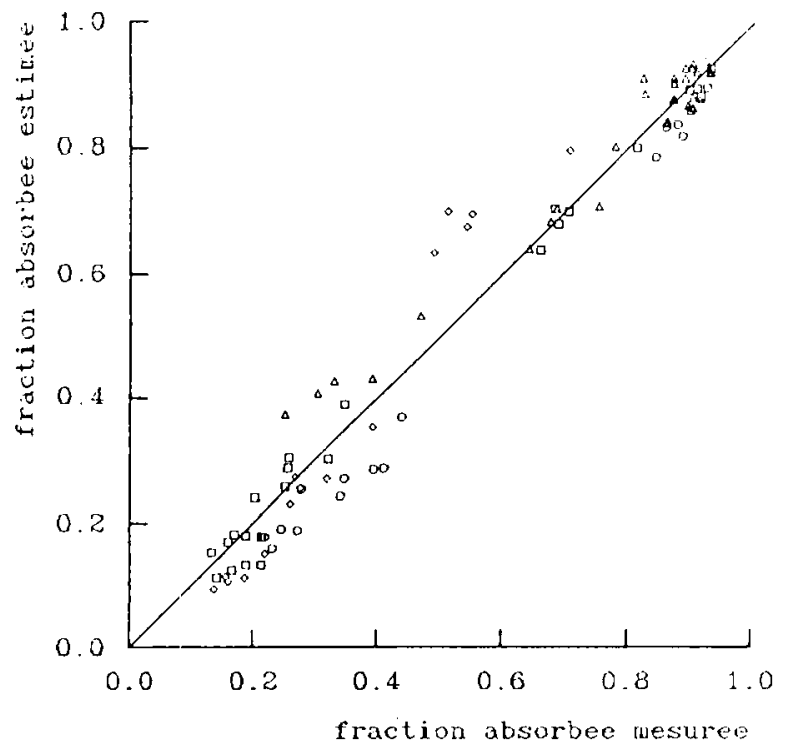

Fig. 8. Relation entre la fraction absorbée mesurée à partir de photographies hémisphériques et la fraction absorbée estimée à partir de l'indice de végétation et du modèle (8). Chaque traitement est représenté par un caractère différent : $\Delta$ : Fidel $1 ; \bigcirc$ : Fidel 2; $\square$ : Arminda 2; $\diamond$ : Fidel 3 . Pour chaque traitement, la fraction absorbée est mesurée dans les 5 conditions d'éclairement spécifiées au paragraphe III-A-3 et estimée à partir des paramètres $K_{\varepsilon i} / K_{D N}$ du tableau 3 (modèle a).

optiques du sol (ou de la strate sénescente) sous-jacent qui peuvent être très variables et difficiles à appréhender. La sensibilité de l'indice de végétation aux propriétés optiques des sols induit une variabilité particulièrement importante de la relation entre $\varepsilon_{i}$ et $\mathrm{DN}$ dans le cas de sols sombres. Le port foliaire participe aussi à la définition de la relation entre $\varepsilon_{i}$ et DN.

L'utilisation de modèles simplifiés décrivant les relations entre $\varepsilon_{i}$ et LAl d'une part et entre DN et LAl d'autre part a permis de formaliser la relation entre $\varepsilon_{j}$ et DN en une expression analytique (équation (8)) prenant en compte explicitement l'effet du sol et ne comportant qu'un seul paramètre $\mathrm{K}_{\varepsilon i} / \mathrm{K}_{\mathrm{DN}}$. Ce paramètre décrit la non linéarité de l'équation (8) et est sous la dépendance des conditions d'éclairement et du port foliaire. Dans certaines conditions particulières, $K_{\varepsilon i} / K_{D N}$ est égal à 1,00 et la relation (8) devient linéaire et conforme aux résultats d'autres auteurs.

L'expérimentation effectuée sur des cultures de blé révèle la justesse de l'approche théorique ainsi que ses limites. L'ajustement du modèle (8) aux données expérimentales mesurées sur des cultures de blé en phase d'installation, montre que le paramètre $K_{\varepsilon i} / K_{D N}$ est significativement supérieur à 1,00 rendant la relation entre $\varepsilon_{i}$ et $D N$ non linéaire. Le paramètre $K_{\varepsilon i} / K_{D N}$ dépend bien de la géométrie d'éclairement. En revanche, il ne nous a pas été possible de mettre en évidence la dépendance de ce paramètre par rapport à l'ar- chitecture du couvert comme le suggérait l'analyse théorique.

L'ensemble de ce travail permet donc d'envisager l'utilisation de la télédétection pour estimer la biomasse produite par les cultures de blé, par le biais d'une estimation de l'énergie photosynthétiquement active absorbée par le couvert. Mais la précision de cette technique dépendra en particulier de la capacité qu'ont les indices de végétation de minimiser l'influence des propriétés optiques du sol. II faudrait également introduire les effets liés à la réponse non linéaire de la photosynthèse à l'éclairement, et améliorer notre connaissance du déterminisme du facteur $\varepsilon_{b}$ de conversion de l'énergie absorbée en biomasse.

Enfin, il conviendra de définir des stratégies d'interpolation entre les dates de mesures satellitaires. La fréquence actuelle de ces mesures (Spot, Landsat) ne permet pas, en effet, un suivi dans le temps de l'évolution de la fraction PAR absorbée compatible avec l'estimation de la biomasse produite par le couvert.

\section{RÉFÉRENCES}

Ajai, Sashikumar M.N., Kamat D.S., Aggarwal P.K. \& Sinha S.K. (1984) Spectral estimates of intercepted photosynthetically active radiation by wheat canopies. Proc. crop growth conditions and remote sensing. 22-23 June 1984, ISRO-IARI, 411-419

Asrar G., Fuchs M., Kanemasu E.T. \& Hatfield J.L. (1984) Estimating absorbed photosynthetic radiation and leaf area index from spectral reflectance in wheat. Agron. J. 76, 300-306

Badhwar G.D., Verhoef W. \& Bunnik N.J.J. (1985) A comparative study of Suits and SAIL canopy reflectance models. Remote Sens. Environ. 17, 179-195

Baret F. (1986) Contribution au suivi radiométrique de cultures de céréales. Thèse université Paris-Sud (Orsay) No 98, 1-182

Baret F. \& Major D. (1988) Significance of the normalized difference. Workshop : Vegetation indices and their interpretation, Nottingham 1988/05/25

Bonhomme R. \& Chartier P. (1972) The interpretation and automatic measurements of hemispherical photographs to obtain sunlit foliage and gap frequency. Israel. J. Agric. Res. 22, 53-61

Bouvier A., Gelis F., Huet S. Messean A. \& Neveu P. (1985) CS-NL (notice détaillée de l'utilisation du logiciel d'ajustement non linéaire CS-NL). Laboratoire de biométrie, INRA-CNRZ, Jouy-en-Josas

Daughtry C.S.T. (1988) Estimating absorbed radiation and phytomass from multispectral reflectance of corn and soybean. Proc. IGARS'88 ESA SP 284, 821-824

Ducrey M. (1975) Utilisation des photographies hémisphériques pour le calcul de la perméabilité des couverts forestiers au rayonnement solaire. I. Analyse théorique de l'interception. Ann. Sci. Forest. 32, 73-92

Ducrey M. (1975b) Utilisation des photographies hémisphériques pour le calcul de la perméabilité des cou- 
verts forestiers au rayonnement solaire. II. Etude expérimentale. Ann. Sci. Forest. 32, 205-221

Garcia R., Kanemasu E.T., Blad B.L., Bauer A., Hatfield J.H., Major D.J., Reginato R.J. \& Hubbard K.G. (1988) Interception and use efficiency of light in winter wheat under different nitrogen regimes. Agric. For. Meteorol. 44, 175-186

Goel N.S. \& Strebel D.E. (1984) Simple beta distribution representation of leaf orientation in vegetation canopies. Agron. J. 76, 800-802

Goel N.S. \& Deering D.W. (1985) Evaluation of a canopy reflectance model for LAl estimation through its inversion. IEEE Trans. Geosci Remote Sens. GE 23, 674-684

Gosse G., Varlet-Granchet C., Bonhomme R., Chartier M., Allirand J.M. \& Lemaire G. (1986) Production maxjmale de matière sèche et rayonnement solaire intercepté par un couvert végétal. Agronomie 6, 47-56

Green C.F. (1987) Nitrogen nutrition and wheat growth in relation to absorbed solar radiation. Agric. For Meteorol. 41, 207-248

Guyot G., Hanocq J.F., Buis J.P. \& Saint G. (1984) Mise au point d'un radiomètre de simulation de Spot. $2^{\circ}$ Col. Int. Signatures Spectrales d'Objets en Télédétection. Bordeaux. 12-16 Sept. 1983. INRA 233-242

Hatfield J.L., Asrar G. \& Kanemasu E.T. (1984) Intercepted photosynthetically active radiation in wheat canopies estimated by spectral reflectance. Remote Sens. Environ. 14, 65-75

Kouassi N. (1986) Suivi d'une culture de blé par télédétection. Mémoires de fin d'études INRA, Montfavet, France

Kumar M. (1988) Crop canopy spectral reflectance. Int. J. Remote Sensing, 9, 285-294
Le Master E.W., Chance J.E. \& Wiegand C.L. (1980) A seasonal verification of the Suits spectral reflectance model for wheat. Photogramm. Eng. Remote sens. 46, 107-114

Monteith J.L. (1972) Solar radiation and productivity in tropical ecosystems. J. Appl. Ecol. 9, 747-766

Monteith J.L. (1981) Does light limit crop production? In : Physiological processes limiting plant production C.B. Johnson (ed.) Butterworths (London) : 23-38

Olioso A. (1987) Utilisation de photos hémisphériques pour étudier l'efficience de la conversion de l'énergie solaire en biomasse par une culture de blé. Mémoire INRA/ENSAM. INRA, Montfavet, France

Rigal D. \& Terres J.M. (1988) Modélisation du profil spectral de cultures de mais et de soja. Estimation de la biomasse produite. Mémoire INRA-ESAP : INRA, Montfavet, France, 1-85

Rouse J.W., Haas R.H., Schell J.A., Deering D.W. \& Harlan J.C. (1974) Monitoring the vernal advancement and retrogradation of natural vegetation. NASA/GSFC. Type III. Final report. Greenbelt MD : $1-371$

Sellers P.J. (1985) Canopy reflectance, photosynthesis and transpiration. Int. J. Remote Sensing. 6, 1335 1372

Varlet-Grancher C., Bonhomme R., Chartier M. \& Artis P. (1982) Efficience de la conversion de l'énergie solaire par un couvert végétal. Acta Oecol. Oecol. Plant. 3(17) 1, 2-26

Verhoef W. (1984) Light scattering by leaf layers with application to canopy reflectance modeling : the SAIL model. Remote Sens. Environ. 16, 125-141

Verhoef W. (1985) Earth observation modeling based on layer scattering matrices. Remote Sens. Environ. 17, 164-178 